\title{
Resting State Neural Networks and Energy Metabolism
}

\author{
Raymond Noack \\ Department of Computer Science \\ 140 Governors Drive \\ University of Massachusetts \\ Amherst MA 01003, USA \\ rnoack@cs.umass.edu
}

\author{
Chetan Manjesh \\ Department of Computer Science \\ 140 Governors Drive \\ University of Massachusetts \\ Amherst MA 01003, USA \\ cmanjesh@cs.umass.edu
}

\author{
Miklos Ruszinko \\ University of Massachusetts \\ Amherst MA 01003, USA \& \\ Renyi Institute of Mathematics \\ Budapest, Hungary \\ ruszinko@umass.edu
}

\author{
Hava Siegelmann \\ Department of Computer Science \\ 140 Governors Drive, University of Massachusetts \\ Amherst, MA 01003, USA \\ hava@cs.umass.edu
}

\author{
Robert Kozma \\ Department of Computer Science \\ University of Massachusetts Amherst, MA 01003 \& \\ Dept. Mathematics, U. of Memphis, TN 38152, USA \\ rkozma@cs.umass.edu
}

\begin{abstract}
The human brain is an energy hungry organ. How that brain manages its energy consumption in maintaining its health and executing sensori-motor and cognitive functions is an important but overlooked research area in contemporary cognitive neuroscience. It is argued here that the principal method whereby the human brain manages its energy utilization is through maintaining a relatively elevated level of activity in what can be referred to as "resting state networks" (RSN). The elevated energy consumption in the human brain's varied RSNs is driven and maintained by a physiological mechanism we call the Frame-Formation Energy Cycle (FFEC). Running the FFEC cycle is metabolically expensive and therefore offers a mechanism to explain the increased energy consumption in human-brain RSNs as compared to regions not involved in such networks.
\end{abstract}

Keywords-Resting State Network (RSN); Amplitude Modulation (AM); Metabolism; Energy Constraint; Glia; Spiking Neural Networks.

\section{INTRODUCTION}

The explosion of data on the functional anatomy of the human brain that neuroimaging research has provided us since the turn of the century has begun to identify collections or networks of regions of the brain that act cooperatively even during restful periods when there is no particularly high cognitive demand placed on those regions. These collections have been referred to as "resting state networks" (RSN) [23]. An RSN is a collection of cooperatively behaving brain regions and is characterized by a high "functional connectivity" between those constituent brain regions relative to what there would be between, say, a collection of random brain regions. Functional connectivity can be defined as the "temporal dependence of neuronal activity patterns of anatomically separated brain regions" identified by "the level of co-activation of functional MRI time-series measured during rest" [23]. Some examples of RSNs in the human brain are the visual network, auditory network, sensorimotor network, default mode network, language network, executive network, and several others [20, 23]. Early concepts of visual receptive fields [13] can be seen as precursors of activity networks as described by RSNs.

As more and more RSNs have been identified over the past 15 years, researchers began to become interested in how the formation and maintenance of these RSNs produce behavior and cognition in animals, especially humans. A similar interest is developing as to how the breakdown in maintenance and interaction of these RSNs may lead to neurodegenerative diseases, such as dementia and schizophrenia [9]. RSNs also help to address issues related to cognitive load and driver fatigue [19]. In both the healthy and diseased brain, the manner in which cortical neurons and networks of neurons utilize the energy resources available to them is of critical importance. Understanding how these networks acquire and metabolize nutrients and discard waste products and excess heat will provide keen insight into how these RSNs are formed and maintained, as well as yield valuable insight into how changes in those processes alter normal brain function.

Since nutrients, $\mathrm{O} 2, \mathrm{CO} 2$, waste products, and heat dissipation are all exchanged through the blood flow in the brain, and since RSNs are largely identified through changes in that blood flow, a good place to start an investigation is to 
look at the BOLD signal that informs the identity of these RSNs [3, 22]. While, historically, there has been a debate on the origins of the BOLD signal, the growing consensus is that they are fundamentally driven through cooperatively behaving populations of neurons in various proximately and disparately connected brain regions both intrahemispherically and inter-hemispherically [22]. The BOLD signal is generated through excessive blood flow (hyperemia) necessary to coordinate cooperative behavior between these local and disparate cortical regions. The excessive blood flow is necessary in the regions constituting the RSNs because a sustained cooperative activity between these regions demands high amount of energy even at rest. This demand is satisfied by the increased cerebral flow to the regions of the RSN in order to deliver an increase in $\mathrm{O} 2$ and glucose to not only the cooperatively interacting neurons, but also to the closely associated glia cells that intimately interact with the neurons in the metabolic cycle $[1,15]$. Furthermore, the increased cerebral blood flow (CBF) supplied to these RSN regions is likely required to remove the excessive $\mathrm{CO} 2$, waste products, as well as dissipate excess heat.

Recently, Marcus Raichle, one of the pioneers of brain research using neuroimaging to probe human brain function, has hypothesized that RSNs serve to manage a sort of "dark energy" in the brain, an energy that binds the regions of a given RSN together [18]. The manifestation of this binding is identified as a tightly organized and specialized cross-talk or "chatter" that goes on between the regions of an RSN and which keeps it in a ready state to respond quickly to either sensory stimuli or internal cognitive signals.

So crucial to understanding the functioning of the human brain are these RSNs, that Raichle put out a call to researchers to begin to build models of how the brain manages this dark energy by specifically highlighting one important RSN in particular, the default mode network (DMN). Raichle states [18]:

"Looking ahead, investigators must now try to glean how coordinated activity among and within brain systems operates at the level of the individual cells and how the DMN causes chemical and electrical signals to be transmitted through brain circuits. New theories will then be needed to integrate data on cells, circuits and entire neural systems to produce a broader picture of how the brain's default mode of function serves as a master organizer of its dark energy. Over time neural dark energy may ultimately be revealed as the very essence of what makes us tick."

In this work we heed Raichle's call and outline a multiscale model for the management of metabolic/energy dynamics within RSN's displaying high functional connectivity. We begin with a brief review of what functional connectivity is and how it identifies the constitution of human-brain RSNs. We follow this review by introducing the Frame Formation Energy Cycle (FFEC), the metabolic cycle we propose to describe the dynamics of interacting populations within and between the constitutive regions of any given $\mathrm{RSN}$ and that gives rise to the enigmatic dark energy. We present a preliminary population model of spiking neurons, including neuron-glia metabolism effects. We conclude with discussions of future research directions.

\section{FUNCTIONAL ANATOMY OF RESTING STATE NETWORKS}

An RSN is a group of structurally and functionally associated brain regions whereby cooperative behavior in the network typically serves to execute some particular class of tasks, including visual recognition, auditory recognition, and language production/recognition $[2,22,23]$. While the constitution of some RSNs makes intuitive sense, the reason for the formations of other RSNs is not so obvious. For example, there is a "primary visual" RSN that connects, bilaterally, the primary visual cortices in the human cortex. In addition, there is an auditory RSN that connects, also bilaterally, human auditory cortices [20]. Less intuitively, however, there are also RSNs composed of what may be considered to be more obscure brain regions. For example, the "default mode network" (DMN), a network thought to be involved in idle thought or daydreaming, is an RSN composed principally of the posterior cingulate cortex, medial prefrontal cortex, medial temporal lobe, precuneus and temporoparietal junction [2]. The "executive network" RSN, on the other hand, includes predominantly the dorsolateral prefrontal cortex and anterior cingulate cortex [20]. The executive network is involved more in targeted tasks such as focused problem solving.

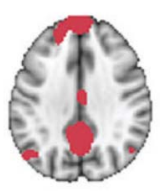

(1)

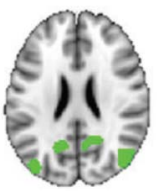

(2)

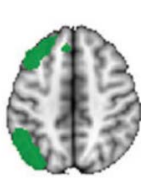

(3)

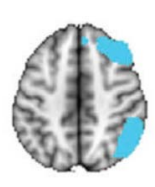

(4)

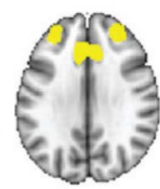

(5)
Fig. 1 The identification of five Resting State Networks (RSNs) in the human brain. From left to right, the 1) dorsal default mode network, 2) ventral default mode network, 3) left executive control network, 4) right executive control network, and 5) anterior salience network [2].

While it makes intuitive sense that cooperatively interacting brain regions such as the visual cortices or auditory cortices would form an RSN, what is especially interesting about the discovery of RSNs like the DMN and executive network is that they inform more non-intuitive notions of how the brain executes it functions, what functions it deems important, and what brain regions are involved in those functions. Another interesting feature of human-brain RSNs is that the functional connectivity among the constitutive regions seems to correlate with a likewise enhanced structural connectivity [2]. Although it is tempting to associate the enhanced structural connectivity between regions of a given $\mathrm{RSN}$ with its enhanced 
functional connectivity $[2,10]$ and simply leave it at that, we believe it is important to investigate more closely how function follows form within the networks, paying particular attention as to how the energy budget of the brain plays a principal role.

\section{THE FFEC AND THE FORMATION AND MAINTAINANCE OF BRAIN RSNs}

In order to understand the origins of the elevated BOLD signals identifying the functional connectivity that defines an RSN, it is necessary to discuss how the populations of neurons that compose the varied regions of the RSN interact. The model we will use here to describe the dynamics of neural populations follows the work of Freeman and Kozma [5, 6, 7, 16], who have developed a model for the production, manipulation, and transmission of perceptual and cognitive frames of experience known as the "cinematic model." Each individual frame in the cinematic model corresponds to a specific percept such as the visual image of an apple or a mental "mind's eye" image of an apple. The formation of temporal sequences of such images and the motor behaviors that accompany them, such as reaching out, grabbing, and taking a bite of an apple, are played out through the temporal sequencing of these frames in the human brain much like the frames in a film reel are played through a projector.

Each of the frames described above are manifested, in the cortex in particular, as a spatial pattern of amplitude modulated (AM) activity in a given cyto-architectonically defined cortical region. These regions, e.g., the primary visual cortex or dorsolateral prefrontal cortex, are the regions that compose a given RSN. These AM patterns are formed in the cortex when data from an external sensory stimuli enter the primary sensory cortices or when an endogenous signal arising within the brain itself, such a cognitive thought, triggers a synaptically coupled or strengthened assembly of neurons, what has been classically referred to as a "Hebbian cell assembly" (HCA) [11].

The AM pattern that emerges from the activation of a given cell assembly manifests a perceptual/cognitive frame and the formation of the AM pattern provides the coherent and coordinated behavior within said cortical area that is necessary to transmit its identity or qualitative character to the other regions within the cortex and brain in general in order to inform the production of an associated AM pattern in that region. The frame formation process occurs through a four-stage cycle, which is referred to as the cinematic cycle of cognitive brain dynamics $[6,16]$ :

\section{Stage 1. "Disordered background" stage}

The firings of individual neurons are relatively uncoordinated with one another resulting in low phase coherence among the population of neurons constituting the region, as well as low analytic amplitude, which is representative of the spatial pattern of activation in the population.
The energy cost in stage one is relatively low due to the sparse, uncorrelated firing of the neurons.

\section{Stage 2. "Trigger" stage}

A Hebbian cell-assembly becomes triggered which is marked by a gain or increase in the firing rate between the constituent neurons in the population due to facilitated synaptic connections. This action creates a well-defined AM pattern, which is reflective of an individual percept or cognitive frame.

This stage of the cycle likely exacts a high-energy cost in that the increased firing rate in the population necessitates utilization of ATP from the Krebs cycle of the mitochondria to run the neurons' sodium-potassium pumps [1]. Such demand likely also drains the glycogen stores in the glia cells (astrocytes) that are closely associated with the neurons in the pool.

\section{Stage 3. "Frame-formation" stage}

The emergence of the AM pattern peaks. This allows the identity of the AM pattern to be sent along convergent and divergent axons to each of the other regions involved in the RSN which, in turn, initiates a stage two process of AM formation in those regions.

Stage three likely exacts a high energy cost for the same reason as described for stage two.

\section{Stage 4. "Dissolution" stage}

The AM pattern disintegrates as new stimuli bombard the population and de-couples the cooperatively interacting neurons. At this point, the population returns to its stage one baseline level of activity, then the cycle repeats.

The energy utilization in stage four is also likely low as CBF serves to restore the glycogen stores, the oxygen debt is "repaid," and ATP production begins to crank up through the Krebs cycle in the mitochondria $[1,15]$.

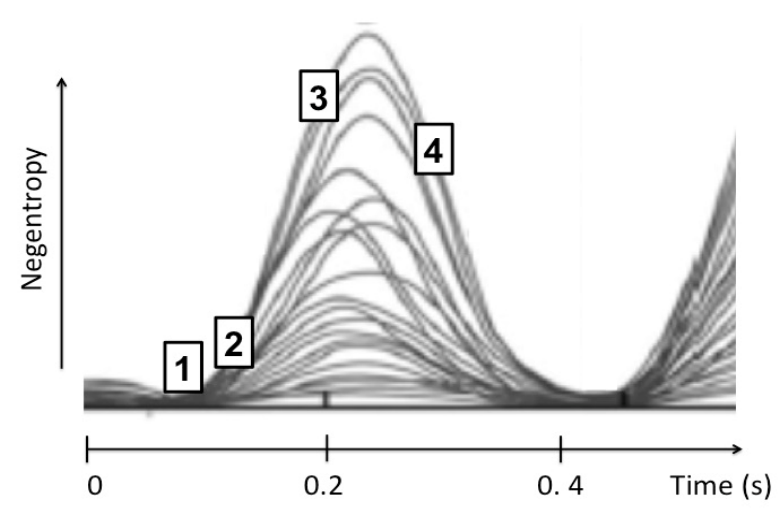

Figure 2. Visualization of the FFEC; (1) disordered background stage; (2) trigger stage; (3) AM pattern formation stage; (4) dissolution (erosion) stage. The negentropy increases with increasing order in the system, as shown here by analytic amplitudes of multichannel EEG data; adopted from [16]. 


\section{METABOLIC MODEL OF RSN FORMATION AND MAINTENANCE}

Functional connectivity is the temporal dependence of neuronal activity patterns between brain regions [23]. We propose that this temporal dependence is provided for by the reinforcement of AM pattern formation between those anatomically separated brain regions. The elevated and highly coordinated BOLD signals that distinguish any given RSN's identity from background brain activity are likely the result of the similarly coordinated timing of the energy demands brought about through the formation of the AM patterns in the FFE cycle described above.

In order to run and maintain the FFEC, certain energy demands must be met for the constituent neural populations. Below we outline a multi-level metabolic model that supports the FFEC and is responsible for the coordinated BOLD signals that inform the functional connectivity that identifies given RSNs.

\section{A. Bi-phasic metabolic cycle of AM patterns}

In describing how this metabolic process proceeds, it is necessary to discuss how incoming signals entering a given neuron populations convert to the outgoing AM patterns that characterize the important contribution of the FFEC to the maintenance of RSNs. Accordingly, we propose the following bi-phasic metabolic approach of resting state brain activity:

\section{Low-activity metabolic state:}

During this state, which corresponds to the dissolution phase and disordered background phase of the FFEC, the population is performing more it's housekeeping functions of paying back it's a) oxygen debt or $\mathrm{O} 2$ replacement, b) $\mathrm{CO} 2$ and waste removal, and c) excessive heat dissipation.

2. High-activity metabolic state:

During this state, which corresponds to the trigger phase and frame formation phase of the FFEC, ATP production is ramped up in the mitochondria of neurons which facilitates the high spiking density of these neurons during AM pattern formation, leading to the depletion of glycogen stores in astrocytes.

\section{B. Large-scale broadcast through RSNs}

Since the BOLD signal fluctuation period is significantly longer than that the FFEC period $(\sim 0.1 \mathrm{~Hz}$ versus $\sim 5 \mathrm{~Hz})$, we argue that the CBF that informs the BOLD signal is best viewed as a mostly a steady reservoir for heat dissipation and nutrient exchange and that the BOLD fluctuations reflect a more smoothed out and generalized oscillation timed by the phase synchrony of shared AM pattern formation between the constitutive regions of any given RSN. RSNs can be considered as plesiochronous entities with metastable dynamics, in which the synchrony continually erodes and ultimately switches to the state with a lack of synchrony.

In our proposed interpretation, AM patterns are viewed as mesoscopic manifestations of cognitive content, which communicate through the RSN during their transmission phase to maintain some specific state. The duration of a FFEC is about $0.2 \mathrm{~s}$, so there are several dozens of such cycles during the underlying RSN BOLD dynamics of $\sim 10$ s. The FFEC provides a mesoscopic link from microscopic neurons to macroscopic networks across the hemispheres.

\section{Multi-level metabolic model of the FFEC}

The proposed model has 3 levels, namely, microscopic, mesoscopic, and macroscopic. The microscopic cellular level is based on the neuron-glia system using the ANLS shuttle [1]; see Fig. 3. The neuron-glia metabolic system is used to provide input to spiking neurons modeled, e.g., by leaky integrate-and-fire (LIF) neurons, or Izhikevich neurons [14]. Pools of excitatory and inhibitory spiking neurons are combined into the mesoscopic level. Mesoscopic populations produce the AM patterns that carry the cognitive content and are measurable by EEG. The mesoscopic AM patterns are connected into RSNs which are measurable via fMRI. Measured and calculated neural signatures are compared and the model is adjusted based on any mismatch. This multi-level metabolic model is illustrated in Fig. 4.

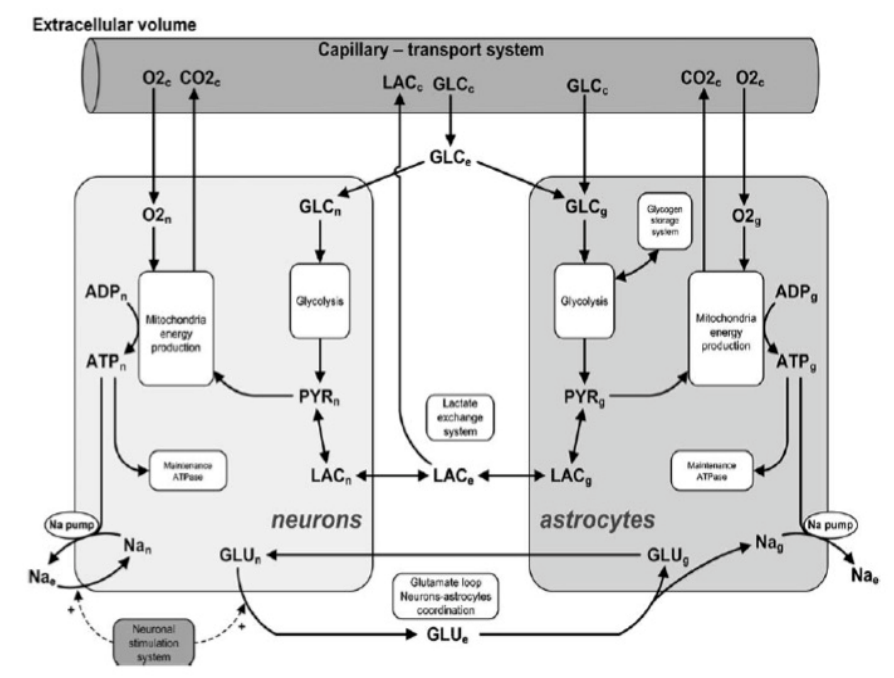

Figure 3. Diagram of the neuron-glia system and astrocyte-neuron lactate shuttle (ANLS), from [1].

It is to be stressed that mesoscopic dynamics manifested in AM patterns is monitored by EEG (scalp) or ECoG (intracranial) arrays, while macroscopic RSNs are accessible by large-scale fMRI monitoring. In the rest of this paper we focus on elaborating a very simplified microscopic model of neuron-glia metabolism feeding to spiking neuron activity in populations of excitatory and inhibitory neurons. In the 
basic cellular metabolic model, no explicit considerations are made on the characterization of calcium dynamics [8]. In a more complete description, the link between mesoscopic EEG/ECoG dynamics and macroscopic BOLD RSNs will be analyzed in future studies.

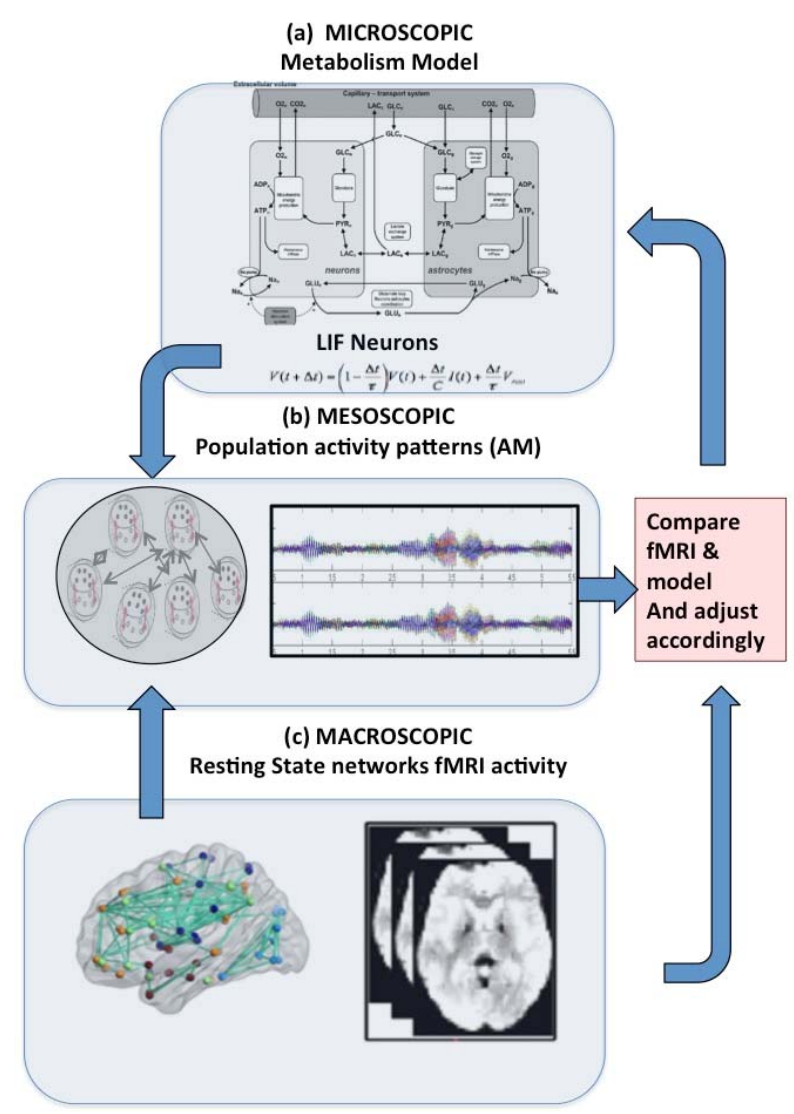

Figure 4. Diagram of the multi-level metabolic model; (a) microscopic level with neuron-glia metabolism and spiking neurons; (b) mesoscopic level with pools of excitatory-inhibitory neurons; (c) macroscopic level with brain-wide RSNs; based on [21].

\section{COMPUTATIONAL SIMULATION OF THE MICROSCOPIC AND MESOSCOPIC LEVELS}

\section{A. Microscopic model of a coupled neuron and glia unit}

As an initial step in the development of the broader multi-level metabolic model, we describe here a simple model to simulate the microscopic dynamics of individual metabolic glia-neuron couplings and their participation in a mesoscopic network. The simulation is based on Izhikevich (IZ) spiking neurons [14]. IZ is a spiking-neuron model that distills the large number of interacting chemical processes inside neurons that act to produce spiking behavior into two differential equations [14]. In a similar fashion, we reduced the roughly 50 differential equations of the Cloutier et al. astrocyte-neuron metabolic model [2] into just two differential equations. The end result is the formation of what we call the "reduced" Cloutier-IZ metabolic model, which is composed of four differential equations, as follows:

$$
\begin{aligned}
& \frac{d g(t)}{d t}=-\tau_{1} g(t)-\frac{\beta_{1}}{c_{1}+m(t)}+\mu_{1} G_{1}(t) \\
& \frac{d m(t)}{d t}=-\tau_{2} m(t)-\frac{\beta_{2}}{c_{2}+g(t)}+\mu_{2} G_{2}(t) \\
& \frac{d v(t)}{d t}=0.04 v^{2}+5 v+140-u+I(t)+\alpha m(t) \\
& \frac{d u(t)}{d t}=a(b v(t)-u(t))
\end{aligned}
$$

Equation 1 represents the rate of glycogen $(\mathrm{g})$ formation and storage in the glia cell that will ultimately be used as a fuel source for the glia cell's accompanying neuron. Equation 2 represents the rate of ATP production in the mitochondria $(\mathrm{m})$ of the neuron. The rate of ATP production is the principle element that binds the two reduced models (Cloutier and IZ) insofar as it places energy constraints on the efficacy of spike production in the IZ simulation/model. Specifically, the IZ spiking model does not account for any anomalies in the efficacy of the sodiumpotassium pump of neurons, which is instrumental in resetting the resting membrane potential of those neurons. Normal spiking behavior is reliant on the efficient operation of this pump, which, in turn, is reliant on the adequate production of ATP in the neuron mitochondria.

The functional utility of Eqs. $1 \& 2$ (reduced Cloutier model) is to model anomalies in the nutrient (glucose) and oxygen availability of the astrocyte-neuron pairs. This availability is represented by the $\mathrm{G}_{1}$ variable in equation one, which represents the glucose availability to the astrocyte (glia), and by $\mathrm{G}_{2}$ in Eq. 2, which represents the combined availability of glucose to the neuron in the presence of oxygen to model oxidative phosphorylation. In Eq. $1 \& 2, \tau_{1}$ and $\tau_{2}$ are time constants of glia and mitochondria dynamics; $\mu_{1}$ and $\mu_{2}$ are amplitude factors for glucose supply; $\beta_{1}$ and $c_{1}$, and $\beta_{2}$ and $c_{2}$ are coefficient in the coupling terms between glia and mitochondria, respectively.

Equations $3 \& 4$ are taken directly from the IZ simple spiking neuron model; for details, see [14]. The two sets of equations (Cloutier and IZ) are coupled together through the " $\mathrm{m}$ " variable in Eq. 3, using coupling parameter $\alpha$. This term adds a condition to the effective operation of the neurons' spiking behavior based on metabolic processes. Eq. 3 for (v) represents the time-varying value of the membrane potential of each individual neuron, while Eq. 4 for $(u)$ represents the manner in which the membrane potential resets itself after the production of a spike and how it provides negative feedback to $\mathrm{v}$ in the effort to maintain a set resting membrane potential, see Fig. 5. 


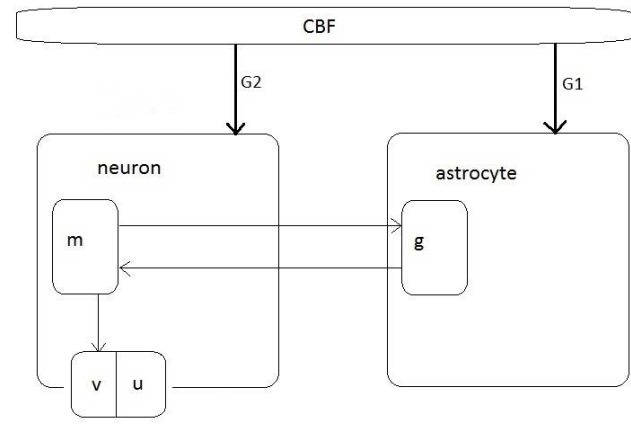

Figure 5. Schematic diagram of the relationship of variables used in the general model in Eqs. 1-4, and in computer simulations; CBF stands for cerebral blood flow. This is simplified version of the model shown in Fig. 3.

\section{B. Mesoscopic metabolic model of excitatory and inhibitory populations}

For the computer simulation, we have coupled 800 excitatory neurons and 200 inhibitory neurons together into a fully interconnected network; each neuron is connected to all the others with a random connection weight. This is described in Eqs. 5-8, where $i$ denotes the $i$-th spiking neuron, $i=1, \ldots, 1000$.

$$
\begin{gathered}
\frac{d g_{i}(t)}{d t}=-\tau_{1} g_{i}(t)-\frac{\beta_{1}}{c_{1}+m_{i}(t)}+\mu_{1} G_{1}(t) \\
\frac{d m_{i}(t)}{d t}=-\tau_{2} m_{i}(t)-\frac{\beta_{2}}{c_{2}+g_{i}(t)}+\mu_{2} G_{2}(t) \\
\frac{d v_{i}(t)}{d t}=0.04 v_{i}^{2}(t)+5 v_{i}(t)+140-u_{i}(t)+I_{i}(t) \\
+\sum_{i=1}^{N} \alpha m_{i}(t)
\end{gathered}
$$

$$
\frac{d u_{i}(t)}{d t}=a\left(b v_{i}(t)-u_{i}(t)\right)
$$

The 4:1 ratio of excitatory neurons to inhibitory neurons corresponds to the functional morphology of the neocortex [14]. The strengths of the weights between excitatory and inhibitory neurons are chosen to produce biologically feasible behavior of the neuron population; for a detailed description, see [14]. At first, we conducted numerical experiments to reproduce the results of the standard IZ simulation. The results of the simulation are shown in Figure 6, representing a normal baseline glucogenic state of the brain metabolics, as expected.

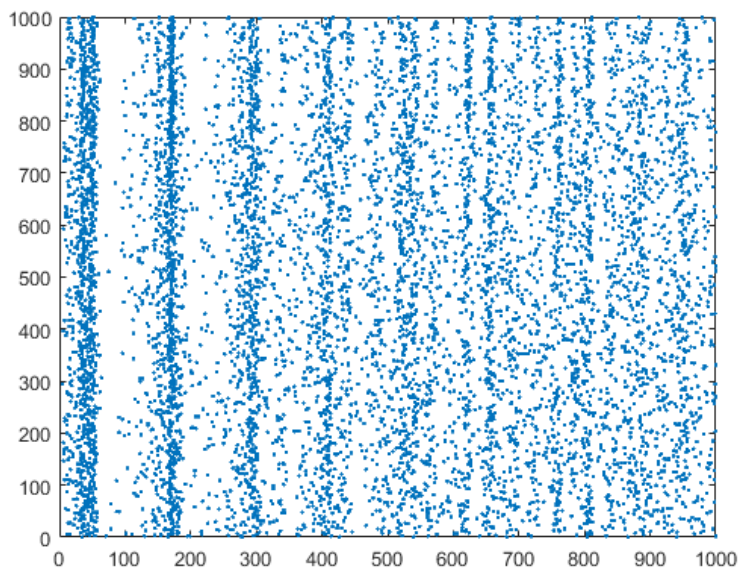

Figure 6. Sample run of the IZ model simulation (without metabolic equations). The vertical axis of the spike raster plot indicates the position of each of the 1000 neurons involved in the network. The horizontal axis represents a one-second epoch divided into a 1000 time steps, each of duration 1ms. Each individual blue dot reflects the neuron firing a spike at the indicated time step.

Next we simulate the metabolic effect on the IZ neuron populations. In these experiments, we fitted the variable $\mathrm{G}_{1}$ to a sine function of varying frequencies up to $100 \mathrm{~Hz}$, while $\mathrm{G}_{2}$ has been implemented as a normally distributed random variable. For simplicity, we set the time constants and other parameters to unity, with the exception of $c_{1}$ and $\mathrm{c}_{2}$, which were set to 2 . Obviously, more detailed parameter studies are to be conducted in future studies.

Results of simulations with two different frequencies are given in Fig. 7 and Fig. 8, respectively. We observe, that depending on the frequency of the driving sine oscillations, the spiking population activity can exhibit strong synchronization effects.
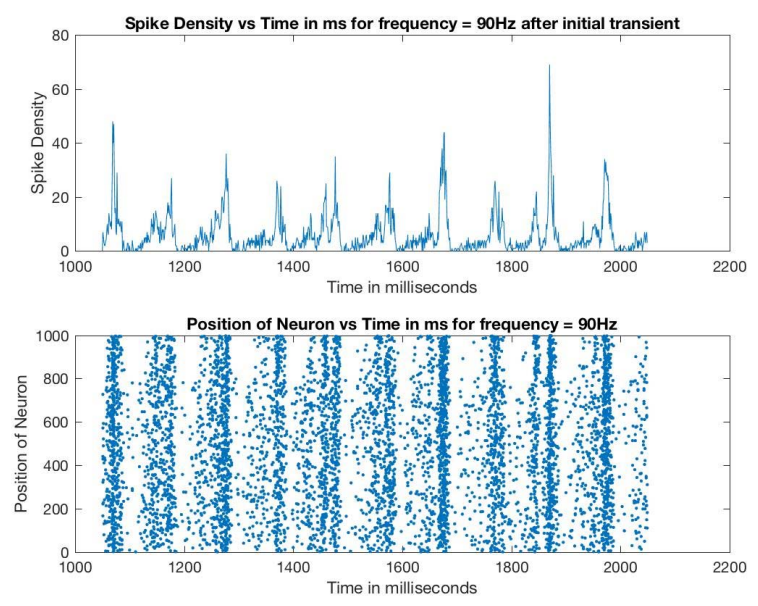

Figure 7. Sample run of reduced IZ-Cloutier simulation, with driving frequency $90 \mathrm{~Hz}$ of the $\mathrm{G}_{1}$ component. Note the similarity in the form of the raster plot to the original IZ simulation. 
In Fig. 7, the driving frequency is $90 \mathrm{~Hz}$, and the oscillations are largely similar to the baseline IZ results shown in Fig. 6. For driving frequency $70 \mathrm{~Hz}$, however, we see strong synchronization at around $10 \mathrm{~Hz}$. The spike density plots shown in Figs. 7 and 8 , reinforce this conclusion, showing that for $\mathrm{f}=70 \mathrm{~Hz}$ in Fig. 8, most of the 1000 IZ neurons contribute to the "beating," while for 90 $\mathrm{Hz}$, only about $40-50$ neurons are in synchrony, that is $4-5$ $\%$ of the total pool.
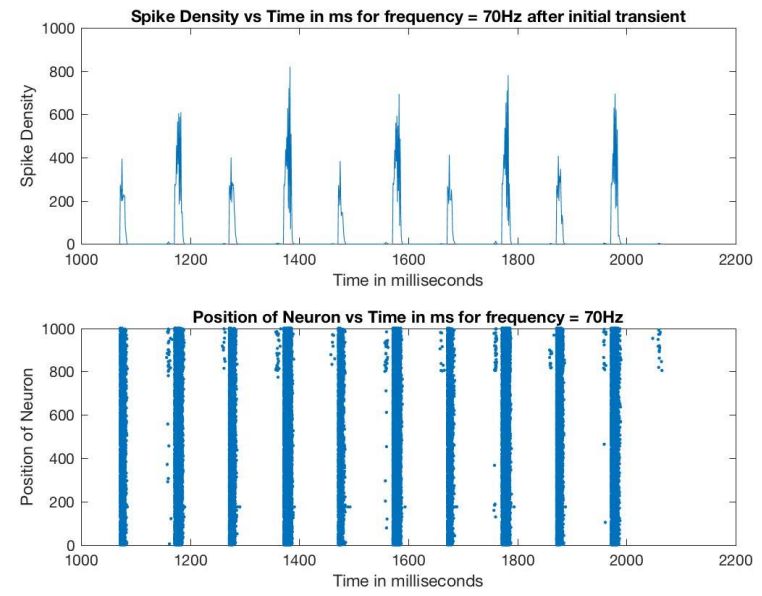

Figure 8. Sample run of reduced IZ-Cloutier simulation, with driving frequency $70 \mathrm{~Hz}$ of the $\mathrm{G}_{1}$ component. Note the appearance of the strong oscillation covering large part of the neural populations; about 400-700 neurons.

\section{CONCLUSIONS AND FUTURE DIRECTIONS}

The establishment of a model for the formation and maintenance of brain RSNs from the perspective of the FFEC is useful because it allows one to investigate how changes in the energy parameters of the system/brain may affect the cycle and thus the formation and interaction of various RSNs.

In this work we present preliminary results with the coupled metabolic and spiking neuron model. In future simulations, we will fine-tune the time and proportionality constants in an effort to simulate varied metabolic states in the human, such as hypoglycemic and ketogenic conditions. This should also allow us to explore how those states may affect the microscopic spiking behavior of neurons and the accompanying mesoscopic effects that that individual microscopic spiking behavior produces. At a longer term, our goal is to couple many of the above-described mesoscopic networks into a larger macroscopic computer model that effectively simulates the macroscopic threetiered metabolic model.

There are many factors that affect these energy parameters such as nutritional factors, neurodegenerative diseases, vascular diseases, etc. Intensive research is conducted on the role of nutritional factors by looking how certain diets that cause ketogenic and hypoglycemic states affect the energy (ATP) production in the mitochondria of glial-neuron complexes relative to a baseline glucogenic state. Certain differences in production rates and availability of energy will certainly affect the sensitive character and timing of the formation of AM patterns within and between regions of a given RSN via a change in the dynamics of the FFEC cycle. It is of interest to explore the way the proposed ANLS (astrocyte-neuron lactate shuttle) and glycogen storage systems $[2,10]$ in astrocytes may provide the extra energy boost necessary to realize the peak AM pattern of the FFEC cycle through the metabolic function of the constitutive glia-neuron networks.

Through such efforts, we are hoping to produce a generalized model that can be used to predict how changes in the energy profile of the brain will affect its functional connectivity under healthy and unhealthy conditions.

Acknowledgments: This work has been supported in part by grants DARPA-HR0011-16-1-0006 and NSF-13-11165.

\section{REFERENCES}

[1] Cloutier, M., Bolger, F.B., Lowry, J.P. and Wellstead, P. 2009. An integrative dynamic model of brain energy metabolism using in vivo neurochemical measurements. Journal of Computational Neuroscience, 27(3), pp.391-414.

[2] Deco, G., Jirsa, V.K. and McIntosh, A.R., 2011. Emerging concepts for the dynamical organization of resting-state activity in the brain. Nature Reviews Neuroscience, 12(1), pp.43-56.

[3] Crossley, N.A, Mechelli, A., Vértes, P.E., Winton-Brown, T.T., Patel, A.X., Ginestet, C.E., McGuire, P., Bullmore, E.T., 2013. Cognitive relevance of the community structure of the human brain functional coactivation network. Proceedings of the National Academy of Sciences, 110(28), pp. 11583-8.

[4] Fox, M.D. and Raichle, M. E., 2007. Spontaneous fluctuations in brain activity observed with functional magnetic resonance imaging. Nature Reviews Neuroscience, 8(9), pp.700-11.

[5] Freeman, W. J., 1975. "Mass Action in the Nervous System," Academic Press, New York.

[6] Freeman, W. J., 2006. A cinematographic hypothesis of cortical dynamics in perception. International Journal of Psychophysiology, 60, pp.149-161.

[7] Freeman, W. J. (2007). Proposed cortical "shutter", mechanism in cinematographic perception. In R. Kozma \& L. Perlovsky (Eds.), Neurodynamics of cognition and consciousness (pp. 11-38). Springer Verlag. New York.

[8] Ghosh, S., \& Basu, A. 2012. Calcium signaling in cerebral vasoregulation. In Calcium Signaling (pp. 833-858). Springer Netherlands.

[9] Haatveit, B., Jensen, J., Alnæs, D., Kaufmann, T., Brandt, C.L., Thoresen, C., Andreassen, O.A., Melle, I., Ueland, T., Westlye, L.T., 2016. Reduced load-dependent default mode network deactivation across executive tasks in schizophrenia spectrum disorders. NeuroImage: Clinical, 12, pp.389-96.

[10] Haimovici, A., Tagliazucchi, E., Balenzuela, P., Chialvo, D. R., 2013. Brain organization into resting state networks emerges at criticality on a model of the human connectome. Physical Review Letters, 110(8101). 
[11] Hebb, D.O. 1949. "The organization of behavior." John Wiley and Sons. New York

[12] Heinonen, J., Numminen, J., Hlushchuk, Y., Antell, H., Taatila, V. and Suomala, J., 2016. Default mode and executive networks areas: association with the serial order in divergent thinking. PLoS One, 11(9), e0162234.

[13] Hubel, D. H., \& Wiesel, T. N. 1962. Receptive fields, binocular interaction and functional architecture in the cat's visual cortex. The Journal of physiology, 160 (1), 106-154.

[14] Izhikevich, E.M., 2003. Simple model of spiking neurons. IEEE Transactions on Neural Networks, 14(6), pp.1569-1572.

[15] Jolivet, R., Coggan, J.S., Allaman, I., Magistretti, P.J., 2015. Multi-timescale modeling of activity-dependent metabolic coupling in the neuron-glia vasculature ensemble. PLoS Computational Biology, 11(2), e1004036.

[16] Kozma, R. and Freeman, W.J., 2016. "Cognitive phase transitions in the cerebral cortex-enhancing the neuron doctrine my modeling neural fields." Springer. New York.

[17] Noack, R.A., 2012. Solving the "human problem": the frontalfeedback model. Consciousness and Cognition, 21(2), pp.1043-67.

[18] Raichle M.E., 2010. The brain's dark energy, Scientific American, 302(3):pp. 44-9.
[19] Ros, T., Baars, B. J., Lanius, R. A., \& Vuilleumier, P. 2014. Tuning pathological brain oscillations with neurofeedback: a systems neuroscience framework. Frontiers in Human Neuroscience, 8, pp. 56-73.

[20] Shirer, W.R, Ryali, S., Rykhlevskaia, E., Menon, V. and Greicius, M.D., 2012. Decoding subject-driven cognitive states with whole-brain connectivity patterns. Cerebral Cortex, 22(1), pp.158-65.

[21] Siegelmann, H. and Mujica-Parodi, L. R., 2015. Individual variability in human brain connectivity modeled using multiscale dynamics under energy constraints. Unpublished manuscript.

[22] Sridharan, D., Levitin, D.J. and Menon, V., 2008. A critical role for the right fronto-insular cortex in switching between central-executive and default-mode networks. Proceedings of the National Academy of Sciences, 105, pp.12569-12574.

[23] Van den Heuvel, M.P. and Hulshoff Pol, H.E., 2010. Exploring the brain network: a review on resting-state fMRI functional connectivity. European Neuropsychopharmacology, 20(8), pp.519-34. 